\title{
Une offensive tous azimuts chez le manufacturier Louis Garneau Sports - LGS
}

\author{
Raymond Poisson \\ Université Laval
}

\section{Introduction}

Depuis quelques années, le secteur manufacturier québécois et canadien dans l'ensemble fait face à deux pressions majeures: la concurrence des producteurs des pays émergents, ainsi que la remontée graduelle mais rapide de la valeur du dollar canadien. L'entreprise qui n'a pas augmenté son niveau de productivité rencontre aujourd'hui un problème important, ses coûts ne lui permettant pas de faire face à la concurrence des produits importés. Le problème peut être moins grave si cette entreprise a adopté une stratégie de différenciation. En effet, si ses produits ont toujours une longueur d'avance en matière d'innovation, elle peut éventuellement s'en sortir. Mais est-ce suffisant? Dans les faits, comment un manufacturier peut-il tirer profitablement son épingle du jeu dans un contexte où les concurrents sont nombreux, où certains bénéficient de coûts de main-d'œuvre ridiculement bas et des compétences pour exporter un produit bien accepté et reçu par les consommateurs. Après une recherche documentaire remontant aux premiers écrits dans la presse concernant le manufacturier Louis Garneau Sports LGS, j'ai eu le plaisir d'avoir trois entrevues avec monsieur Sylvain Boudreault, directeur général, pour tenter de répondre à cette question. Dans cet article, le lecteur trouvera certains éléments lesquels, à mon avis, expliquent en bonne partie le succès de cette PME.

\section{LGS, d'hier à aujourd'hui}

L'entreprise, sise à Saint-Augustin-de-Desmaures dans le comté de Portneuf, a pris son envol en 1983. Après treize ans comme coureur cycliste international, Louis Garneau, appuyé de son épouse Monique Arsenault, entreprend la confection de ses premiers vêtements cyclistes dans le garage paternel. Un coup de pouce de $6000,00 \$$ de sa grand-mère beauceronne, ainsi que l'aide occasionnelle de ses parents pour la fabrication, permet de concrétiser le projet d'entreprise. En 1984, après avoir participé aux Jeux olympiques de Los Angeles, le propriétaire dirigeant décide de quitter la compétition active à titre de cycliste et de s'orienter vers le monde des affaires. De quelques vêtements fabriqués lors des premiers mois, l'entreprise prend rapidement de l'expansion car ses produits sont bien accueillis sur le marché. En 1988, Sylvain Boudreault entre en scène chez LGS à titre de directeur général, dans la foulée de cette croissance des affaires.

LGS, c'est le cœur de ce qu'il serait juste d'appeler le Groupe Louis Garneau et Monique Arsenault. L'entreprise possède trois usines dont deux sont à

Saint-Augustin-deDesmaures. Y est effectuée la fabrication de vêtements techniques ${ }^{1}$ : de la création du modèle jusqu'à l'assemblage final des pièces, en passant par l'impression par sublimation ou par thermo-transfert sur les vêtements, et la coupe. LGS fut d'ailleurs la première à introduire en Amérique du Nord ce procédé d'impression. L'entreprise possède également une filiale de fabrication à Newport, dans l'État du Vermont (LG-USA). Celle-ci effectue de l'assemblage de pièces coupées expédiées directement des usines de la maison-mère deux fois par semaine. LGS peut compter sur deux autres usines appartenant à autant de compagnies différentes détenues par Monique Arsenault. Sécurité Sport fabrique des casques de vélos; quant à Confection WG, elle fait de l'assemblage de vêtements. Sylvain Boudreault souligne qu'en 1988, LGS pouvait compter sur 2972 mètres carrés d'espace de production; en 2006, on parle de près de 23700 mètres 
carrés. En plus des cinq usines détenues directement ou indirectement, LGS a des accords de production avec trois compagnies sous-traitantes.

Fin 2005, LGS est une entreprise qui affiche des ventes totales de l'ordre de 62 millions de dollars; environ $69 \%$ de ses ventes provient du Canada, 25 $\%$ des États-Unis et $6 \%$ de l'Europe, de l'Asie et de l'Amérique du sud. Fabriquant uniquement des vêtements pour les cyclistes initialement, l'entreprise a évolué. Sur son site web (www.louisgarneau.com), elle est définie comme manufacturier et distributeur de produits sportifs haut de gamme, orientée principalement vers les vêtements, casques et accessoires de vélo, vêtements et accessoires de ski de randonnée et de plein air, casques de ski alpin, vêtements pour enfants, vêtements personnalisés pour équipes et entreprises, vêtements multisports, ainsi que vélos et appareils de conditionnement physique.

\section{Le paysage au sein duquel évolue le coureur}

Le noyau des affaires ou core business de l'entreprise, c'est le vêtement. Or, l'industrie du textile et du vêtement est durement frappée par la mondialisation des marchés depuis plusieurs années. L'Accord Multifibres a pris fin le 1er janvier 2005; cet accord protégeait les marchés des pays industrialisés de l'invasion des produits des pays à bas coûts de main-d'œuvre. Les vêtements fabriqués en Asie inondent les marchés. La Chine et l'Inde sont les pays qui font les plus importantes percées, tout particulièrement vers les États-Unis, principal marché extérieur de LGS. Entre 1997 et 2004, les exportations de vêtements de la Chine sont passées de 7 milliards de dollars US à 13 milliards de dollars US, soit un taux de croissance annuel composé de 9,2\%. L'Inde connaît une croissance de ses exportations vers le marché américain de l'ordre de 10 $\%$ par année, textiles et vêtements confondus. Au Canada, les importations de vêtements en provenance de la Chine ont quadruplé entre 1992 et 2004 et le mouvement s'accentue.

Contrairement au secteur du textile, celui du vêtement est exigeant en main-d'œuvre pour la fabrication. La délocalisation des activités de fabrication devient donc une solution qui s'impose de plus en plus, aux yeux des entreprises des pays industriali- sés, pour demeurer compétitif sur les marchés ${ }^{2}$. LGS sent la pression monter pour faire de même. De façon générale cependant, le secteur du vêtement évolue pour faire face à la concurrence étrangère. De nombreux manufacturiers investissent dans la mécanisation et l'informatisation des équipements de production. On constate une évolution technologique dans ce secteur. Conséquemment, selon le Conseil canadien des ressources humaines de l'industrie du vêtement (CRHIV), l'industrie devrait avoir un grand besoin de personnes capables de travailler avec des outils de coupe très perfectionnés, comme le laser ou l'infrarouge; ces gens devront connaître l'ensemble des opérations d'assemblage de vêtements. Des gens formés dans la logistique, les technologies de l'information et le contrôle de la qualité, ainsi que des techniciens de bon niveau, seront également requis par les entreprises manufacturières de l'industrie du vêtement. Par contre, deux phénomènes conjugués risquent de ralentir le développement du secteur : un taux élevé de roulement des employés et le vieillissement de la main-d'œuvre actuelle. La main-d'œuvre spécialisée ou qualifiée se fait rare. Quand l'économie va bien, plusieurs secteurs industriels sont en concurrence pour attirer des employés chez eux : le secteur du vêtement est donc en compétition pour le renouvellement ou l'ajout de main-d'œuvre ${ }^{3}$.

Enfin, le marché au sein duquel évolue LGS est relativement restreint et la concurrence y est vive. Selon Sylvain Boudreault, le marché cycliste canadien serait de l'ordre de 200 millions de dollars; côté ski de fond, on parle de 100 millions de dollars environ. Le marché américain est dix fois plus grand, mais la compétition y est féroce. Dans le marché des casques, par exemple, les Bell Sport, Specialized, Trek et plusieurs autres se battent pour des parts de marché. Dans le vêtement, Pearl Izumi est un compétiteur majeur aux États-Unis et il n'est pas le seul; au Canada, Sugoi de Vancouver est très présente et il y en a d'autres. Depuis peu, la compagnie œuvre également dans le domaine des vélos de route et des vélos stationnaires d'exercice. Encore là, la compétition est forte.

Que fait LGS pour se développer dans un environnement globalement hostile, pour un marché somme toute très pointu? 


\section{Une direction organisée orientée vers le futur}

Dès 1988, Louis Garneau était conscient que la croissance de son entreprise requérait la présence d'un gestionnaire. Il a donc embauché Sylvain Boudreault à titre de directeur général. Détenteur d'une maîtrise en finance, celui-ci amenait ses connaissances et ses compétences en gestion. Dès lors, un partage des responsabilités a été effectué et surtout, respecté. Comme le dit monsieur Boudreault :

Louis, ce qu'il a fait de très intéressant, c'est qu'il a su utiliser le potentiel et la force des employés. Louis, c'est un génie créateur. Il pond des idées et il est très fort en idées, en marketing; il est très bon en gestion aussi mais il a su se spécialiser dans sa force qui est le design et la création. Parallèlement, moi j'ai développé une équipe de directeurs de gestion. [...] Moi, je ne touche pas au design; lui, je dirais qu'il me laisse le champ libre pour la gestion, les opérations, l'ouverture des usines, les acquisitions, l'expansion.

\section{Afin de gérer le plus adéquatement à court comme à moyen et long terme, LGS peut compter sur trois lieux de réflexion.}

Cette gestion partagée a comme catalyseur d'action une seule et même préoccupation, selon Sylvain Boudreault : "Nous, ce qui nous inquiète, ce n'est pas 2005-2006, c'est 2008-2009. Il faut se préoccuper d'être rentable dans ce temps-là. Le marché va s'orienter comment? » Peu de choses sont laissées au hasard chez LGS. Les dirigeants savent où ils veulent mettre le focus, ce qu'ils doivent privilégier comme produits, comme approche de mise en marché, comme investissement en équipement et machinerie. Telle ou telle pièce n'est pas nécessaire aujourd'hui, mais elle le sera dans deux ans: l'entreprise investit pour le futur en achetant maintenant. Afin de gérer le plus adéquatement à court comme à moyen et long terme, LGS peut compter sur trois lieux de réflexion.

Tout d'abord, tous les lundis matin, il y a une réunion des directeurs de service avec le président et le directeur général pour faire le point. Le directeur de la filiale Chlorophylle, ainsi que celui de LGUSA, sont en communication téléphonique. C'est l'occasion d'analyser ce qui s'est passé la semaine précédente et de planifier la semaine courante. Annuellement, Louis Garneau préside une rencontre d'orientation de deux jours, une rencontre de " vision" selon le terme de Sylvain Boudreault. Le groupe de direction établit à cette occasion les grandes orientations pour l'entreprise. Quelles sont les grandes tendances du marché? Quelle ligne de produits doit être développée? Devons-nous en délaisser? Si oui, lesquels? Et ce ne sont pas là les seules questions. Enfin, LGS peut compter sur l'appui d'un comité consultatif mis sur pied en 1989. Ce comité est composé de sept personnes. Deux personnes sont de LGS, soient Louis Garneau et Sylvain Boudreault. Les cinq autres sont des gens travaillant dans différentes sphères d'activités. Il y a un avocat et un industriel très connu au Québec dans le domaine du vêtement. On retrouve aussi quelqu'un de leur cabinet comptable, de même qu'un consultant qui fait de la réorganisation, du marketing, puis des mandats de redressement. Une personne spécialisée dans le capital de risque apporte également une expérience très riche au sein du comité.

De ce qui précède ressort un certain nombre de points importants à retenir. Tout d'abord, Louis Garneau a eu la sagesse de reconnaître ses propres limites, ainsi que ses forces. C'est d'abord et avant tout un entrepreneur. Un gestionnaire était nécessaire pour la réalisation et le développement de ses projets et il est passé à l'action pour en trouver un. De plus, le partage des responsabilités est clair, sans ambiguïté, sans chevauchement et il est respecté par les deux hommes. Les forces vives ont donc été canalisées de façon la plus optimale possible, les employés n'étant pas aux prises avec des instructions ou des requêtes contradictoires. Dans la foulée du point précédent, Louis Garneau a été capable de considérer Sylvain Boudreault comme un alter ego, même s'il n'est pas copropriétaire de l'entreprise. Comme ce dernier me le disait, il est capable de discuter avec le président de LGS de façon franche et ouverte, ce qui permet de prendre des décisions après un examen de la situation sans ménagement, sans sollicitude. Les deux hommes forment un solide tandem comme le souligne monsieur Boudreault. L'expérience humaine et professionnelle vécue entre eux depuis 1988 s'est traduite par une remarquable croissance de l'entreprise. De 
3 millions de dollars de chiffre d'affaires en 1988 , LGS a atteint 62 millions de dollars en $2005(+18 \%$ / année). Tout propriétaire dirigeant d'entreprise ou lecteur averti dirait que le défi est de choisir le bon gestionnaire. Mais là, il n'y a pas de recette magique pour ce faire. À la base toutefois, le propriétaire dirigeant doit être un joueur d'équipe afin d'être en mesure d'effectuer un partage des responsabilités et surtout, de le respecter.

Deux autres éléments sont à retenir comme facteurs contribuant à la réussite de l'entreprise. Tout d'abord, il y a la reconnaissance de l'importance de la planification, qu'elle soit stratégique ou opérationnelle, malgré un environnement changeant. Les dirigeants déterminent le cap tout en étant conscient que des vents contraires peuvent les forcer à corriger la trajectoire pour se rendre à destination. Ensuite, l'ouverture d'esprit du propriétaire dirigeant joue un rôle non négligeable : nous ne sommes pas en face d'un one man show. Cette ouverture se manifeste par la consultation des employés clés, de même que par le recours à un comité consultatif constitué principalement de membres externes œuvrant dans différentes sphères d'activités. Ainsi, la direction bénéficie de plusieurs perspectives pour analyser une situation, ainsi que de sources d'information comportant un riche potentiel. Tout ceci concourt à une meilleure qualité de planification, à de meilleurs choix en matière de stratégie.

\section{Une stratégie d'affaires réfléchie fondée sur l'écoute de la clientèle}

LGS a adopté une stratégie de différenciation dans le haut de gamme technique. Monsieur Boudreault précise que l'entreprise va plus loin en visant spécifiquement des niches peu intéressantes pour les grands compétiteurs. De cette façon, elle limite son risque d'affaires en ne mettant pas les pieds sur le marché d'entreprises beaucoup plus puissantes qu'elle. Au Canada, LGS a toutefois décidé depuis longtemps d'offrir une gamme assez étendue de produits de haut de gamme, principalement dans les secteurs suivants: le cyclisme; les sports d'hiver (ski de fond et raquettes de marche); les vélos (de route et d'exercice stationnaire). Notre interlocuteur précise que l'équipe de direction s'assure toujours de viser un marché comportant assez de potentiel pour LGS, mais très peu pour les grandes entreprises.

Lorsqu'il est question des marchés étrangers, la stratégie devient très pointue et ça rapporte des dividendes selon le directeur général. Aux États-Unis, principal marché extérieur de LGS, l'entreprise connaît un bel essor depuis 2002, malgré des débuts modestes en 1989 . Il est question de $20 \%$ à $25 \%$ de croissance par année. Comme le dit Sylvain Boudreault, "On est passé de connu à reconnu ». C'est le fruit d'une stratégie de double focalisation sur le marché. Tout d'abord, LGS a décidé de viser le marché cycliste uniquement. C'est un marché dix fois plus gros que le marché canadien selon lui. De plus, l'entreprise a choisi une niche bien particulière: celle des commandes personnalisées ou commandes d'équipes :

\section{Lorsqu'il est question des marchés étrangers, la stratégie devient très pointue}

Les commandes d'équipes [...] c'est un groupe d'individus qui sont spécialisés dans un sport. Mettons que vous, vous faites du vélo; vous avez une équipe de vélo, vous êtes 15 personnes, vous faites du vélo trois fois par semaine et vous faites 8000 kilomètres par année. [...] c'est des spécialistes. Ce qu'ils veulent, c'est le meilleur du produit, le plus technique, le plus confortable, adapté à leurs particularités d'exercice du sport, qui sont maniaques un peu.

Ces gens achètent donc des vêtements sur mesure, avec leur logo d'équipe. Comme leurs commanditaires peuvent être différents d'une année à l'autre, il y a une certaine récurrence dans les commandes: couleurs et logos changent ainsi que la mode. Grâce à son système de production unitaire, LGS est capable de répondre à des commandes aussi faibles que 16 unités et elle les rentabilise. L'entreprise gère entre 75 et 100 commandes particulières par semaine. Se trouvent parmi ces acheteurs des noms prestigieux tels Harvard University, Massachusetts Institute of Technology - MIT, ainsi que de nombreuses autres universités. LGS vend aussi dans les magasins spécialisés et non seulement à des équipes de cyclistes. Selon Sylvain Boudreault, LGS est une 
alternative intéressante par rapport aux multinationales.

\section{LGS peut compter sur deux sources pour sa veille technologique et commerciale.}

Pour gagner, il faut cependant rester éveillé et à l'affût des commentaires des utilisateurs, comme des tendances ou des goûts régionaux. Les dirigeants de LGS sont très sensibles aux commentaires des utilisateurs et aux demandes du marché. Comme le souligne le directeur général de LGS à titre d'exemple, l'Ouest américain, c'est différent de l'Est. Ainsi en est-il d'autres régions des Etats-Unis ${ }^{4}$. Afin de s'adapter aux particularités des différents marchés du grand ensemble américain, LGS peut compter sur deux sources pour sa veille technologique et commerciale. Tout d'abord, les quelques 2000 clients américains et les 23 agents manufacturiers avec lesquels l'entreprise fait affaire, peuvent leur rapporter les particularités du marché, les orientations, les besoins. Une deuxième source importante de renseignements, c'est la clientèle d'équipes de cyclistes. Parce qu'ils sont des mordus de leur sport, ils n'hésitent pas à souligner à LGS des modifications souhaitables pour améliorer leurs produits. Ce sont des alliés précieux en matière de recherche et de développement car ils sont très exigeants, selon monsieur Boudreault. Toutefois, ces derniers sont des ambassadeurs de choix pour la marque LGS car ce sont des spécialistes, très souvent des équipes professionnelles. Visibilité et crédibilité sont apportées. L'exemple américain en matière de veille est le reflet de ce qui se passe tant au Canada qu'ailleurs dans le monde.

En résumé, LGS a choisi une seule et unique stratégie concurrentielle : la différenciation dans des niches d'activités bien choisies. Ce faisant, l'entreprise est en droite ligne avec ce que Michael Porter ${ }^{5}$ a suggéré voici plus de 20 ans maintenant : elle ne tente pas de vendre des produits novateurs aux prix les plus bas sur le marché, ce qui mène plus souvent qu'autrement à un cul-de-sac au plan de la rentabilité de l'entreprise. De plus, cette stratégie de différenciation prend appui sur un intérêt marqué accordé aux commentaires des utilisateurs de ses produits et des agents manufacturiers qui représentent LGS. Une telle veille contribue à des innovations susceptibles de rencontrer du succès sur les marchés. De plus, cela lui permet de personnaliser son offre de produits et services et de fidéliser sa clientèle.

\section{Une approche de production et des outils à la fine pointe}

Pour survivre et se développer, LGS se bat avec l'arme de l'innovation comme aime à le souligner son président. Cette stratégie ne s'applique pas qu'à la conception des produits, elle s'applique également à la production. Les secteurs du textile et du vêtement sont en régression d'une façon importante au Canada à cause de la concurrence asiatique; LGS a mis en œuvre une stratégie pour s'adapter à un environnement hostile. Conscient que l'entreprise ne peut concurrencer sur tous les fronts avec les produits importés d'Asie, les gestionnaires ont choisi une stratégie de production dite «hybride». Monsieur Boudreault explique de quoi il retourne :

[...] ce qu'il faut faire, c'est utiliser une stratégie basée sur les avantages de l'Asie et les avantages du Canada. Au Canada, ce qui est avantageux, ce sont les petites quantités et les délais de livraison courts. Nous, on s'est spécialisé dans ça. On est maintenant capable de sortir un vêtement en quelques semaines, ce que l'Asie n'est pas capable de faire. On est capable de traiter une commande de 12 ou 18 ou 24 unités, ce que l'Asie n'est pas encore capable de faire. Je ne dis pas que dans 20 ans ou 10 ans ou 5 ans, ils ne seront pas capables de le faire; mais présentement pour eux, c'est un embourbement et non un avantage, parce que ce sont toutes des usines de 1000,2000 et 3000 employés. Il y en a des usines avec moins de main-d'œuvre, mais elles sont plutôt rares.

Bref, lorsqu'une commande arrive chez LGS, les responsables de la production vont analyser certains paramètres pour prendre une décision quant au lieu de fabrication. Lorsque le produit comporte une faible teneur de main-d'œuvre, on a une tendance à produire au Canada. Selon les calculs que les dirigeants ont effectués, le seuil de décision quant au lieu de fabrication d'un vêtement est de l'ordre de 20 minutes de travail : au-dessous de ce seuil, LGS privilégiera la fabrication locale; au-dessus, c'est 
l'Asie qui sera envisagée. À ce paramètre s'ajoutera ceux de la quantité requise et du délai de livraison exigés par le client. L'entreprise a organisé ses sites de production afin de répondre de façon très rentable à des commandes de petites quantités, livrables rapidement.

Monsieur Boudreault souligne que l'inventaire est le talon d'Achille d'un manufacturier de biens influencés par la mode : «Si tu livres un maillot de bain en mars-avril, tu as 100 cennes dans la piastre. Si tu le livres au mois de juillet, ils te demandent un escompte. Et si tu arrives au mois d'août, c'est la désuétude qui rentre, c'est 50 cents dans la piastre, le même maillot de bain. » Il est donc très important pour LGS d'avoir un système de prévision des besoins très raffiné. Il faut commander les bons produits, à la bonne quantité, pour recevoir le tout à temps en fonction des saisons de vente des produits en question. Il faut comprendre que même avec 20 $\%$ ou $30 \%$ du placement complété, LGS passera déjà des commandes de fabrication en Asie à partir d'une extrapolation, d'un estimé des commandes finales. Le risque financier est important car dans son secteur d'activité, tout est affaire de mode. Si l'entreprise fait une erreur d'estimation en plus ou en moins, elle perd de l'argent. Si elle ne commande pas suffisamment d'unités de tel produit, des ventes sont perdues et un client, quelque part, est insatisfait; si LGS surestime les besoins, elle se retrouve avec un surplus d'inventaire qui tombe très rapidement en désuétude. Donc, il ne faut pas se tromper dans les prévisions: il ne faut pas qu'il reste de produits finis à la fin de l'année.

Chez LGS, la gestion de ceux-ci est effectuée de manière très serrée, grâce à un système informatisé de gestion permettant un suivi permanent. Conséquence, la désuétude des produits finis est de l'ordre de $1 \%$, ce qui serait exceptionnel dans ce secteur d'activité, selon monsieur Boudreault. À ce système s'ajoute le Garneau Information Network, lequel est un outil favorisant les ventes tout comme un meilleur contrôle de l'inventaire. Il s'agit d'un extranet permettant à ses clients canadiens et américains de savoir, en temps réel, si tel ou tel produit est en inventaire chez LGS. Par exemple, un client est dans un magasin un samedi matin et veut un vêtement LG taille large non disponible sur place : l'employé peut consulter l'inventaire de LGS et commander immédiatement le vêtement en question s'il est dis- ponible. Le lundi, l'entreprise le livre par l'intermédiaire d'un des transporteurs privés avec lesquels elle a des contrats. LGS bénéficie d'un meilleur prix grâce à de telles ententes et elle en fait bénéficier ses clients, car les frais de transport sont à la charge de ce dernier. Ce système de commerce électronique interentreprises (B2B) a été implanté aux États-Unis en 2002 et deux ans plus tard au Canada.

Pour LGS, se tenir à la fine pointe en matière d'équipement de production est un "must». Lors de nos rencontres, le directeur général est revenu très souvent sur ce point. Une anecdote est assez révélatrice de son credo sur ce point. En fin d'une rencontre, je lui ai fait remarquer à la blague que son bureau était meublé et décoré bien humblement; tout en m'informant que Louis Garneau était du même avis et en faveur d'une rénovation, monsieur Boudreault m'a fait remarquer que ce type de dépense n'était pas un investissement productif. Il préfère de beaucoup acheter en lieu et place une machine à coudre très performante. Une telle approche est sans aucun doute la façon de se distinguer des concurrents, d'aller chercher un avantage compétitif. L'argent est attribué là où ça compte vraiment, comme notre interlocuteur nous le démontre.

Lorsque l'occasion se présente, monsieur Boudreault invite le directeur de la production et son chef mécanicien à partir avec lui pour se rendre à une exposition de matériel de production, peu importe où dans le monde. Ainsi, il peut obtenir l'avis de ceux qui sont directement concernés par l'évolution des équipements de fabrication afin de prendre une décision d'achat. Ce type d'événement leur permet également d'effectuer du «benchmarking " : où se situe LGS en la matière par rapport à ce qui se fait ailleurs. Même si aucun achat n'est réalisé, une telle visite permet de ramener éventuellement des idées pour améliorer le système de production en place. Monsieur Boudreault est conscient qu'un bon arrimage entre l'approvisionnement et la production est source de gains financiers non négligeables. C'est pourquoi en 1999-2000, il a effectué un réaménagement complet des lieux suite à la construction d'un entrepôt semi-automatisé de 31000 pieds carrés. Tout a été pensé pour éviter les pertes de temps et assurer une cadence optimale de la production. 
En somme, trois éléments clés sont associés au volet production. Tout d'abord, le succès repose sur la reconnaissance précise des opportunités et des menaces de l'Asie en la matière. Les gestionnaires se sont livrés à des calculs précis permettant de cerner quand il est rentable de faire fabriquer en Asie ou, inversement, de produire localement. Bref, ils ont agi pour tirer profit d'une situation à première vue défavorable pour eux: la concurrence asiatique. Deuxièmement, l'entreprise a mis au point un système de gestion de l'inventaire à la fine pointe de la technologie. Les dirigeants ne se sont pas contentés d'être conscients que l'inventaire était le talon d'Achille de leur domaine d'activité (reconnaissance d'une menace); ils sont passés à l'action en investissant pour bien contrôler ce vecteur de pertes potentielles (création d'une force). Enfin, ils investissent régulièrement dans leur appareil productif afin d'une part, d'être en mesure de matérialiser les innovations issues de leurs créateurs et, d'autre part, de produire avec qualité, rapidité et au meilleur coût possible.

\section{Une considération marquée pour les em- ployés}

Louis Garneau est fier de ses racines. Cette fierté se traduit aussi par la poursuite d'un objectif : produire le plus possible au Québec, malgré des conditions de marché qui pourraient décourager un moins compétitif que lui. Sylvain Boudreault souligne qu'il y a chez Louis Garneau un engagement moral, en quelque sorte, envers les personnes qui travaillent à Québec : celui de tout faire pour conserver les emplois ici en demeurant compétitif. Le défi est donc de bien agencer la fabrication locale et étrangère, d'être imaginatif pour tenter non seulement de maintenir à tout le moins le niveau de main-d'œuvre locale, mais aussi d'avoir des emplois avec des tâches valorisantes pour les personnes qui les occupent. C'est une préoccupation chez LGS selon notre interlocuteur.

Une visite des lieux permet de constater que les employés bénéficient de certains avantages favorables tant pour eux que pour l'entreprise. Une salle d'exercice avec douches et tout le confort nécessaire est à leur disposition; Sylvain Boudreault souligne qu'elle est très fréquentée. Une cafétéria sur les lieux de travail permet aux employés de manger un repas complet à un prix très raisonnable. Pour déterminer les prix, l'entreprise tient compte du pouvoir d'achat de ses employés qui gagnent le moins, afin qu'ils puissent avoir accès à un repas de qualité. La cafétéria est opérée par une compagnie spécialisée dans ce domaine. Enfin, le vendredi aprèsmidi est un congé pour les employés afin qu'ils puissent bénéficier de cette période pour leurs différents rendez-vous personnels. Cela permet une meilleure conciliation travail/famille pour les employés. L'entreprise y trouve son compte car la chaîne de production risque peu d'être ralentie ou perturbée par l'absence d'un employé clé. LGS tire profit d'un tel arrangement mais les employés ne sont pas en reste, pouvant compter sur une fin de semaine de deux jours et demi. La production s'étale donc sur quatre jours et demi pour un total de 38,75 heures par semaine. Selon monsieur Boudreault, employeur et employés sont très heureux d'un tel arrangement. Dans un proche avenir, Louis Garneau veut installer un centre de création, à côté de la salle d'entraînement installée dans les usines de SaintAugustin-de-Desmaures. Selon sa vision des choses, ce serait un lieu «d'inspiration un peu zen avec des livres sur l'art, un aquarium, un bon système de son, où mes employés pourront aller se ressourcer, se détendre ou encore organiser des réunions. " ${ }^{6} \mathrm{Il}$ s'agit d'une stratégie de R\&D gagnante pour l'employeur et ses concepteurs et chefs de produits, lesquels pourront bénéficier de bonnes conditions pour l'exercice de leur travail de création.

\section{En somme, trois éléments clés sont asso- ciés au volet production.}

\section{Conclusion}

Ce qui a permis à LGS de rester en affaires et de croître est la dynamique complexe entre plusieurs facteurs, dont les principaux éléments sont ceux que j'ai passés en revue dans cet article : une direction organisée orientée vers le futur; une stratégie d'affaires réfléchie fondée sur l'écoute de la clientèle; une approche de production et des outils à la fine pointe; une considération marquée pour les employés. Ces caractéristiques sont-elles novatrices en tant que telles? Sans ambages, je répondrais non. Nombre d'articles scientifiques ou professionnels, 
de livres, font état de ces must en affaires. Il y a cependant tout un monde entre ce qui devrait être en théorie et ce qui est réellement dans les faits. Le cas LGS est un exemple concret d'une réussite due aux qualités de gestionnaire des principaux dirigeants, jumelées à une bonne dose d'esprit entrepreneurial.

\section{Pour faire face tout spécialement au défi asiatique, la stratégie de LGS comportait six volets}

Quelques mois après les entrevues réalisées avec monsieur Sylvain Boudreault, Louis Garneau indiquait à un journaliste que pour faire face tout spécialement au défi asiatique, la stratégie de LGS comportait six volets : valoriser la marque, augmenter le service lié au produit, augmenter la qualité du soutien technique, renforcer le service après vente, contrôler leur réseau de distribution et être à l'affût des besoins des clients ${ }^{7}$. L'établissement de ces actions spécifiques montre que les gestionnaires de l'entreprise ne baissent pas les bras et vont de l'avant, malgré un environnement d'affaires difficile, hostile. L'attitude fait la différence!

\section{Notes et références}

$1 \quad$ Vêtements fabriqués à partir de tissus dits « techniques ». Ces tissus perfectionnés offrent, par exemple, une protection thermique, un plus grand confort pour les activités sportives, une plus grande durabilité.

2 Wyman, D. (2005). Extension ou contraction? Les industries du textile et du vêtement au Canada. Statistique Canada, mars, no. 11-621-MIF2005022.

3 L'habillement, un secteur en pleine restructuration (24 janvier 2004). La Presse, p. 6.; Faire la mode à Québec (11 octobre 2005). Le Soleil, p. B1.

4. http://www.mdeie.gouv.qc.ca/page/web/portail/exportation/ nav/marches_vises/fiches_marches/46404. Accès le 12 décembre 05 .

5 Porter, M. E. (1985). Competitive Advantage: Creating and Sustaining Superior Performance. New-York : Free Press.

$6 \quad$ Innover, une question d'attitude (14 mai 2005). Les Affaires, p. 28.

7 Quatre stratégies de riposte à la concurrence chinoise (28 octobre 2006). Les Affaires, p. 7. 